\title{
Level Fluctuations and Many-Body Effects in Disorder-Free Quantum Dots
}

\author{
Min-Chul Cha ${ }^{1}$ and S.-R. Eric Yang ${ }^{2,3}$ \\ 1)Department of Physics, Hanyang University, Ansan 425-791, Korea \\ 2)Department of Physics, Korea University, Seoul 136-701, Korea \\ 3)Asia Pacific Center for Theoretical Physics, Seoul, Korea
}

\begin{abstract}
We have investigated whether many-body effects can induce significant level fluctuations in a disorder-free quantum dot. The closed energy shell structures and relaxation of the Hartree-Fock (HF) potentials are found to play a significant role. The level degeneracy consistent with the rotational symmetry of the confining potential determines the structure of the energy shells. A closed shell state of a dot can give rise to large fluctuations. When a strong magnetic field is present the shell structure is absent and fluctuations are significantly reduced.
\end{abstract}

PACS numbers: 73.20.Dx, 73.20.Mf

Non-integrable mesoscopic systems exhibit Wigner-Dyson energy level statistics that can be described by random matrix theory [1]. Recently the ground state energy of quantum dots is found to display fluctuations considerably larger than those predicted by random matrix theory [2 5]. The interplay between disorder and electron-electron interactions is expected to be important in explaining the experimental data [5 [8]. However, a deep understanding of this problem is still lacking.

In a disorder-free dot single-particle effects will not induce any fluctuations, and it is usually tacitly assumed that level fluctuations are significantly large only in disordered systems. Whether adding many-body interactions in a disorder-free mesoscopic system will produce observable fluctuations remains an open question. This raises the fundamental question of what type of many-body effects can give rise to significant level fluctuations in a quantum dot. Investigation of such fundamental problems may deepen our understanding the level fluctuations of quantum dots.

In this paper we propose such a many-body effect. In a disorder-free quantum dot new energy shells can exist that are unrelated to the accidental degeneracy of the harmonic potential [9]. The energy shell structure is determined by the degeneracy reflecting the rotational symmetry of the confining potential, and will be present in any size of dot. Because of 2D rotational symmetry of a quantum dot the degeneracy of each shell is significantly reduced compared to that of a real atom. The ordering of energy shells in the dot does not follow a simple rule, and gives rise level fluctuations. The physical origin of this effect is the relaxation of the HF potential, which is omitted in the constant interaction model. When an extra electron is added to the system the other electrons must readjust (relax) their positions self-consistently to minimize the total energy. The new self-consistent potential may be significantly different from the old one, and, therefore, energy ordering may change. We find that this effect can lead to significant level fluctuations. These mechanisms for fluctuations are consequences of the interplay between the shape of the confining dot potential and many body effects.

Our calculation is based on a Hartee-Fock approximation at zero temperature. The HF single particle basis vectors [10] are chosen to be the eigenstates of a quantum dot in a magnetic field described by the Hamiltonian

$$
H_{0}=\frac{\mathbf{p}^{2}}{2 m^{*}}+\frac{1}{2} m^{*} \omega^{2} r^{2}-\frac{1}{2} \omega_{c} \mathbf{L} \cdot \hat{\mathbf{B}}-g \mu_{B} \mathbf{S} \cdot \mathbf{B},
$$

where $m^{*}, B, \omega_{c}$ and $\omega$ are the effective electron mass, magnetic field perpendicular to the 2D plane, the cyclotron frequency, and the effective frequency $\omega^{2}=\omega_{0}^{2}+\frac{1}{4} \omega_{c}^{2}$. A vector potential in a symmetric gauge is used here. The operators $\mathbf{L}$ and $\mathbf{S}$ denote angular momentum and spin of an electron. The eigenstate wave functions of this Hamiltonian are labelled by orbital, angular momentum, and spin quantum numbers $n, l, s$ :

$$
\phi_{n l s}(\mathbf{r})=\frac{1}{\sqrt{2 \pi} a} e^{-i l \theta} R_{n l}\left(\frac{r^{2}}{2 a^{2}}\right) \chi_{s}
$$

with

$$
R_{n l}(x)=\sqrt{\frac{n !}{(n+|l|) !}} e^{-x / 2} x^{\frac{|l|}{2}} L_{n}^{|l|}(x) .
$$

$\chi_{s}$ is spin wave function and the constant $a^{2}=\hbar /\left(2 m^{*} \omega\right)$. The eigenenergies are 


$$
\epsilon_{n l s}^{0}=\hbar \omega(2 n+|l|+1)-\frac{1}{2} \hbar \omega_{c} l-g \mu_{B} S_{z} B
$$

Note that in the presence of a magnetic field $\epsilon_{n l s}^{0} \neq \epsilon_{n l-s}^{0}$.

The electron-electron interaction is given by

$$
V\left(\mathbf{r}_{i}, \mathbf{r}_{j}\right)=\frac{e^{2}}{\kappa \sqrt{\left|\mathbf{r}_{i}-\mathbf{r}_{j}\right|^{2}+d^{2}}},
$$

where $\kappa$ is the dielectric constant and $d$ is the thickness of the planar quantum dot. The HF Hamiltonian has a block diagonalized form. Each block may be labelled by $[l, s]$ and it has the following form

$$
D_{n n^{\prime}}^{[l, s]}=<n l s\left|H_{0}\right| n^{\prime} l s>+<n l s\left|V_{H}\right| n^{\prime} l s>-<n l s\left|V_{X}\right| n^{\prime} l s>.
$$

In order to find the matrix elements of the Hartree $\left(V_{H}\right)$ and exchange $\left(V_{X}\right)$ potentials we need to write HF eigenstates as a linear combination of the HF basis vectors

$$
\left|\alpha l s>=\sum_{m} c_{m}^{(\alpha l s)}\right| m l s>
$$

Invoking rotational invariance about the z-axis we may take the HF single-particle eigenstates, $|\alpha l s\rangle$, to be eigenstates of the z-component of angular momentum operator: $L_{z}|\alpha l s>=\hbar l| \alpha l s>$, where $\alpha$ denotes HF orbital quantum numbers. We find

$$
\begin{array}{r}
<n l s\left|V_{H}\right| n^{\prime} l s>=\sum_{\alpha, p, s^{\prime}}<n l s ; \alpha p s^{\prime}|V| n^{\prime} l s ; \alpha p s^{\prime}>f_{\alpha p s^{\prime}} \\
=\sum_{\alpha, p, s^{\prime}, m, m^{\prime}} c_{m}^{*\left(\alpha, p, s^{\prime}\right)} c_{m^{\prime}}^{\left(\alpha, p, s^{\prime}\right)}<n l s ; m p s^{\prime}|V| n^{\prime} l s ; m^{\prime} p s^{\prime}>f_{\alpha p s^{\prime}}
\end{array}
$$

and

$$
\begin{gathered}
<n l s\left|V_{X}\right| n^{\prime} l s>=\sum_{\alpha, p}<n l s ; \alpha p s|V| \alpha p s ; n^{\prime} l s>f_{\alpha p s} \\
=\sum_{\alpha, p, m, m^{\prime}} c_{m}^{*(\alpha, p, s)} c_{m^{\prime}}^{(\alpha, p, s)}<n l s ; m p s|V| m^{\prime} p s ; n^{\prime} l s>f_{\alpha p s} .
\end{gathered}
$$

where $f_{\alpha p s}$ are the Fermi functions. Note also that even in the absence of a magnetic field the HF method allows partially spin-polarized solution. In such a many-body state $\alpha l+$ and $\alpha l-$ need not be degenerate. For a given number of electrons $N=N_{+}+N_{-}$, the numbers of spin-up $\left(N_{+}\right)$and -down $\left(N_{-}\right)$electrons of the ground state is determined by selecting $\left(N_{+}, N_{-}\right)$which gives the lowest value of ground state energy $E\left(N_{+}, N_{-}\right)$. In such a state the Fermi levels for spin-up and -down electrons match.

As in atomic physics it is possible to introduce energy shells in a quantum dot. However, in a quantum dot the degeneracy of a shell is at most two, consisting of states with two z-component of angular momentum quantum numbers $l$ and $-l$. A shell is thus denoted by $\alpha|l| s$ with $\alpha=1,2, \ldots$ and $|l|=s, p, d, f, \ldots$. The two angular momentum states in a shell may not always be degenerate. It is degenerate only when the total $L_{z}^{\text {tot }}=0$. Our HF calculation shows that states with $L_{z}^{t o t} \neq 0$ can have lower energy than states with $L_{z}^{\text {tot }}=0$. The total $L_{z}$ is zero when there are equal number of occupied states with positive and negative signs of angular momenta below the Fermi level for spin $s$. The degeneracy is lifted when this number is unequal. In such a case the exchange self-energies of the states $\mid \alpha, l, s>$ and $\mid \alpha,-l, s>$ are unequal because the exchange field couples states with opposite signs of angular momentum differently: $\left.\left\langle\alpha, l, s\left|V_{X}\right| \alpha, l, s\right\rangle \neq<\alpha,-l, s\left|V_{X}\right| \alpha,-l, s\right\rangle$.

Like in a real atom the energy ordering of shell energies does not follow a simple rule. The physical origin of this effect is the relaxation of the HF potential. If the single particle wave functions in the $(N+1)$ - and $N$ - particle systems are the same (no relaxation) energy ordering will be unchanged. However, as $N$ changes a new level may appear below the Fermi level: For $N=8$ the spin-up (-down) ground configuration is $1 s^{1} 1 p^{2} 1 d^{2}\left(1 s^{1} 1 p^{2}\right)$. When an extra electron is added a new level $2 \mathrm{~s}$ appears in the spin-up configuration $1 s^{1} 1 p^{2} 2 s^{1} 1 d^{2}$.

The interval between conductance peaks [11] is given by

$$
\Delta_{N}=\mu_{N+1}-\mu_{N}=E_{N+2}+E_{N}-2 E_{N+1} .
$$


where the chemical potential $\mu_{N}=E_{N+1}-E_{N}$ and $E_{N}$ is the total ground state energy as a function of $N$. $\Delta_{N}$ is plotted as a function of $N$ in Fig.1, where $\hbar \omega_{0}\left(\equiv \epsilon_{0}\right)=1 \mathrm{meV}, \kappa=12.4, d=200 \AA$. We remark the following features. In the absence of a magnetic field $\Delta_{N}$ alternate between a trough and a peak as a function of $N$. However, at $N=13,14$ and $N=16,17$ this trend is broken and the values of $\Delta_{N}$ are almost unchanged. The alternation of a trough and a peak reflects closed- and open-shell structures. For $N=12$ the lastly occupied spin-down shell is $1 d^{1}$, which is open (see Fig.2(a)). For $N=13$ the lastly occupied spin-up and -down shells are is $1 f^{2}$ and $1 d^{2}$, which are both closed (see Fig.2(b)). When the system is in a closed shell state $\Delta_{N}$ is larger than the average value while when it is in a partially filled shell $\Delta_{N}$ is smaller. It is difficult to predict when $L_{z}^{\text {tot }}=0$ as a function of $N$ since the HF potential relaxes in a subtle way as an extra electron is added. The variance of $\Delta_{N}$ is $0.31 \hbar \omega_{0}$.

In the presence of a magnetic field $(B=1 T) \Delta_{N}$ exhibits a smooth dependence on $N$, in contrast to the case of no magnetic field. This different dependence on $N$ is due to the breaking of the rotational symmetry by the magnetic field, i.e. the absence of closed shells. The occasional interruption of the smooth dependence of $\Delta_{N}$ on $N$ is due to spin flipping: For small $N$ Coulomb interaction dominates, and, consequently, to minimize the interaction energy, electrons tend to spin polarize. As $N$ increases the confinement energy dominates over the Coulomb energy and it is energetically favorable to pack electrons closely. This state is achieved by flipping a spin and placing it at the center of the quantum dot 12]. The numbers of spin-up and -down electrons are given in Table I for different values of $N$.

We have also investigated the effect of random disorder. The random potential is described by $H_{i m p}(\mathbf{r})=$ $V_{0} \sum_{\alpha} \delta\left(\mathbf{r}-\mathbf{r}_{\alpha}\right)$ where $\mathbf{r}_{\alpha}$ are the positions of 400 randomly located impurities in the area of $\pi\left(12 \sqrt{\hbar / 2 m^{*} \omega_{0}}\right)^{2}$, and $\left|V_{0}\right|=1.5 \mathrm{meV}$ with randomly chosen sign. Figure 1 displays $\Delta_{N}$ and Table I shows the results of $\left(N_{+}, N_{-}\right)$for the ground states. Most of these results can be understood by invoking closed shell structures and relaxation of the HF potentials. However, due to the presence of disorder the degree of spin polarization in $\left(N_{+}, N_{-}\right)$as a function of $N$ is changed in a subtle way.

The main result of our work is that even in a disorder-free dot many-body effects can induce significant level fluctuations. Large level fluctuations do not seem to be a unique signature of a disordered dot. The proposed mechanisms for level fluctuations may serve as a starting point for the understanding level fluctuations of disordered dots.

The work has been supported by the KOSEF under grant 981-0207-085-2, and by Korea Research Foundation under grants 1998-015-D00128 and 1998-015-D00114.

[1] M. L. Mehta, Random Matrices, Academic Press (1991).

[2] R. C. Ashoori, Nature(London) 379, 413 (1996).

[3] A. M. Chang et al., Phys. Rev. Lett. 76, 1695 (1996).

[4] J. A. Folk et al., Phys. Rev. Lett. 76, 1699 (1996).

[5] U. Sivan et al., Phys. Rev. Lett. 77, 1123 (1996).

[6] Ya. M. Blanter, A. D. Mirlin, and B. A. Muzykantskii, Phys. Rev. Lett. 78, 2449 (1997).

[7] R. Berkovits and B. L. Altshuler, Phys. Rev. B 55, 5297 (1997).

[8] H. Tamura and M. Ueda, Phys. Rev. Lett. 79, 1345 (1997).

[9] We only consider dots whose confinement potential is sufficiently weak such that the magic degeneracy of harmonic potential $(2,6,12,20, .$.$) are lifted by electron-electron interactions.$

[10] Our basis set consists of the 120 lowest energy states of a non-interacting single-particle.

[11] In HF approximation the fluctuation is given by $\Delta_{N}=\epsilon_{N+1}^{(N+2)}-\epsilon_{N}^{(N+1)}$ where $\epsilon_{i}^{(j)}$ is the HF single particle energy of eigenstate $j$ when $i$ electrons are present. If the HF potential does not relax then $\epsilon_{i}^{(j)} \approx \epsilon_{i-1}^{(j)}+e^{2} / C$ and $\Delta_{N} \approx$ $\epsilon_{N+1}^{(N+2)}-\epsilon_{N}^{(N+1)}+e^{2} / C\left(e^{2} / C\right.$ is the charging energy $)$.

[12] S.-R. Eric Yang, A.H. MacDonald, and M.D. Johnson, Phys. Rev. Lett. 71, 3194 (1993).

FIG. 1. Level spacing plotted as a function of electron number. Upper (lower) curves show results in the absence (presence) of disorder. The lower curves are shifted vertically for the sake of display. The peaks in $\Delta_{N}$ reflect closed shell states of the dot. 
FIG. 2. Schematic energy diagrams for $N=12$ and 13 (Energy is measured in units of $\epsilon_{0}=1 \mathrm{meV}$ ). Unlike a real atom the maximum number of electrons that a level of a quantum dot can have is two. Note that for $N=12$ spin-down (-up) shells $p$ and $d$ are non-degenerate (degenerate). The explanation of this effect is given in the text. As $N$ changes from 12 to 13 the ordering of $2 s^{1}$ and $1 d^{2}$ shells in the spin-up band is reserved while in the spin-down band split $1 p$ states become degenerate.

TABLE I. The number of $\operatorname{spin}-u p\left(N_{+}\right)$and $-\operatorname{down}\left(N_{-}\right)$ electrons for $\epsilon_{0}=1 \mathrm{meV}$. In the absence of a magnetic field the dot becomes spin-unpolarized as the electron number increases. In the presence of a magnetic field the dot is almost completely spin-polarized. For other values of $\epsilon_{0}$ the degree of spin polarization is different.

\begin{tabular}{ccccc}
\hline \hline & $\begin{array}{c}B=0 \mathrm{~T}^{\mathrm{a}} \\
\left(N_{+}, N_{-}\right)\end{array}$ & $\begin{array}{c}B=1 \mathrm{~T}^{\mathrm{a}} \\
\left(N_{+}, N_{-}\right)\end{array}$ & $\begin{array}{c}B=0 \mathrm{~T}^{\mathrm{b}} \\
\left(N_{+}, N_{-}\right)\end{array}$ & $\begin{array}{c}B=1 \mathrm{~T}^{\mathrm{b}} \\
\left(N_{+}, N_{-}\right)\end{array}$ \\
\hline 1 & $(1,0)$ & $(1,0)$ & $(1,0)$ & $(1,0)$ \\
2 & $(1,1)$ & $(2,0)$ & $(1,1)$ & $(2,0)$ \\
3 & $(3,0)$ & $(3,0)$ & $(3,0)$ & $(3,0)$ \\
4 & $(3,1)$ & $(4,0)$ & $(3,1)$ & $(4,0)$ \\
5 & $(4,1)$ & $(5,0)$ & $(3,2)$ & $(5,0)$ \\
6 & $(5,1)$ & $(6,0)$ & $(3,3)$ & $(5,1)$ \\
7 & $(4,3)$ & $(7,0)$ & $(6,1)$ & $(6,1)$ \\
8 & $(5,3)$ & $(8,0)$ & $(6,2)$ & $(8,0)$ \\
9 & $(6,3)$ & $(9,0)$ & $(6,3)$ & $(9,0)$ \\
10 & $(7,3)$ & $(10,0)$ & $(6,4)$ & $(10,0)$ \\
11 & $(8,3)$ & $(11,0)$ & $(6,5)$ & $(11,0)$ \\
12 & $(8,4)$ & $(12,0)$ & $(6,6)$ & $(12,0)$ \\
13 & $(8,5)$ & $(13,0)$ & $(7,6)$ & $(13,0)$ \\
14 & $(8,6)$ & $(14,0)$ & $(8,6)$ & $(14,0)$ \\
15 & $(8,7)$ & $(15,0)$ & $(9,6)$ & $(15,0)$ \\
16 & $(10,6)$ & $(16,0)$ & $(10,6)$ & $(15,1)$ \\
17 & $(10,7)$ & $(17,0)$ & $(10,7)$ & $(16,1)$ \\
18 & $(10,8)$ & $(17,1)$ & $(10,8)$ & $(17,1)$ \\
19 & $(12,7)$ & $(18,1)$ & $(12,7)$ & $(18,1)$ \\
20 & $(12,8)$ & $(19,1)$ & $(12,8)$ & $(19,1)$ \\
21 & $(13,8)$ & $(20,1)$ & $(11,10)$ & $(20,1)$ \\
22 & $(12,10)$ & $(20,2)$ & $(12,10)$ & $(20,2)$ \\
23 & $(12,11)$ & $(21,2)$ & $(12,11)$ & $(21,2)$ \\
24 & $(12,12)$ & $(22,2)$ & $(12,12)$ & $(22,2)$ \\
25 & $(13,12)$ & $(23,2)$ & $(13,12)$ & $(23,2)$ \\
26 & $(14,12)$ & $(23,3)$ & $(14,12)$ & $(24,2)$ \\
27 & $(16,11)$ & $(24,3)$ & $(15,12)$ & $(25,2)$ \\
28 & $(16,12)$ & $(25,3)$ & $(14,14)$ & $(25,3)$ \\
29 & $(15,14)$ & $(26,3)$ & $(15,14)$ & $(26,3)$ \\
\hline \hline
\end{tabular}

${ }^{a}$ without disorder

${ }^{\mathrm{b}}$ with disorder 


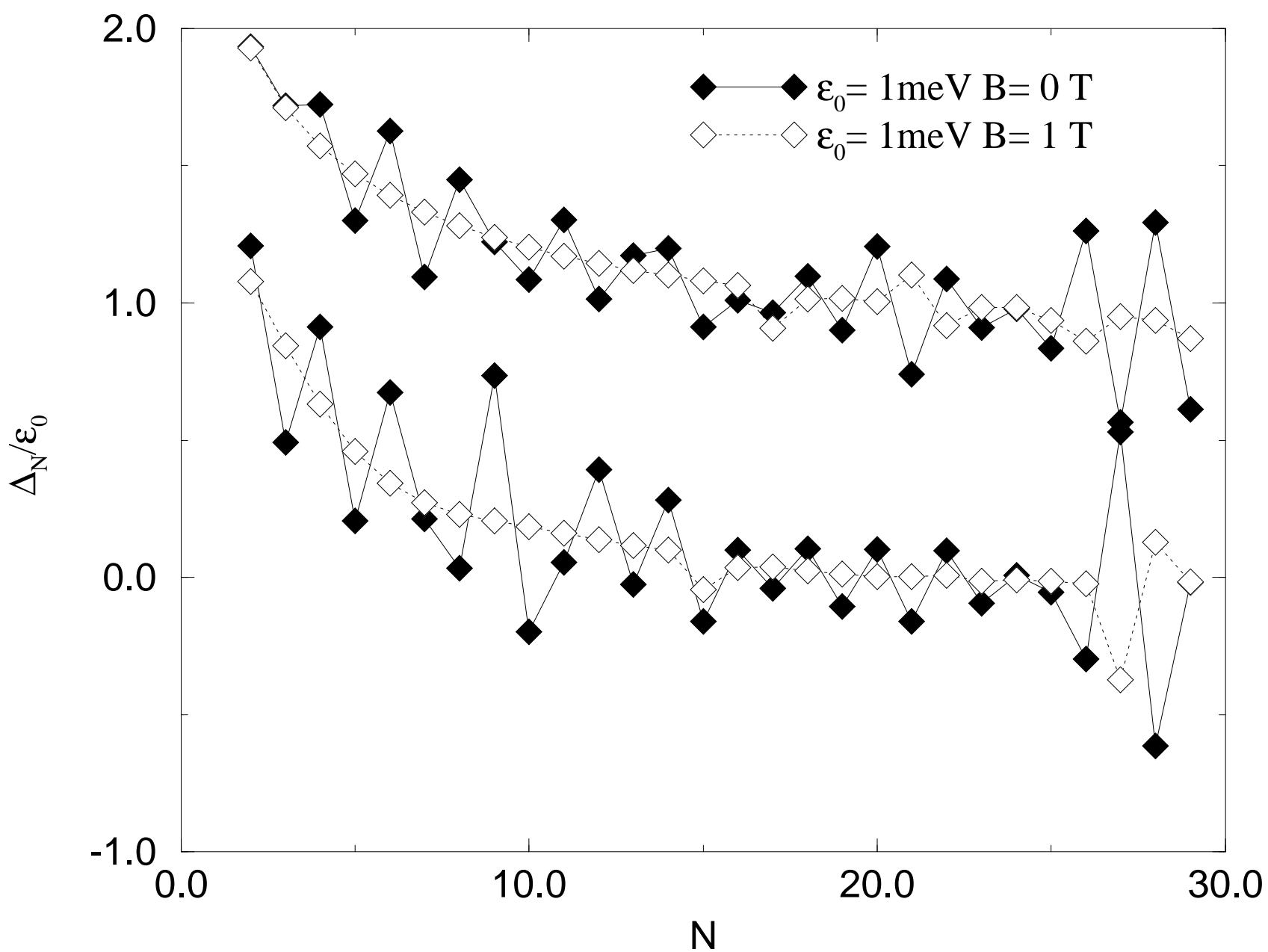


$17.90-1 f^{2}$

$$
17.21={ }_{10}^{2} \mathrm{~s}^{1}
$$$$
16.70-1 p^{2}
$$$$
16.40 \varlimsup_{\text {spin-up }} 1 \mathrm{~s}^{1}
$$
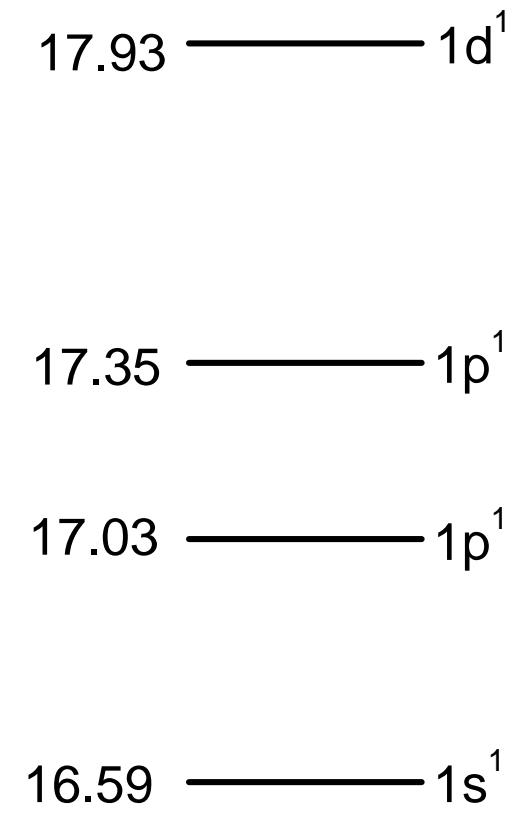

spin-down

$(\mathrm{N}=12)$

(a) 

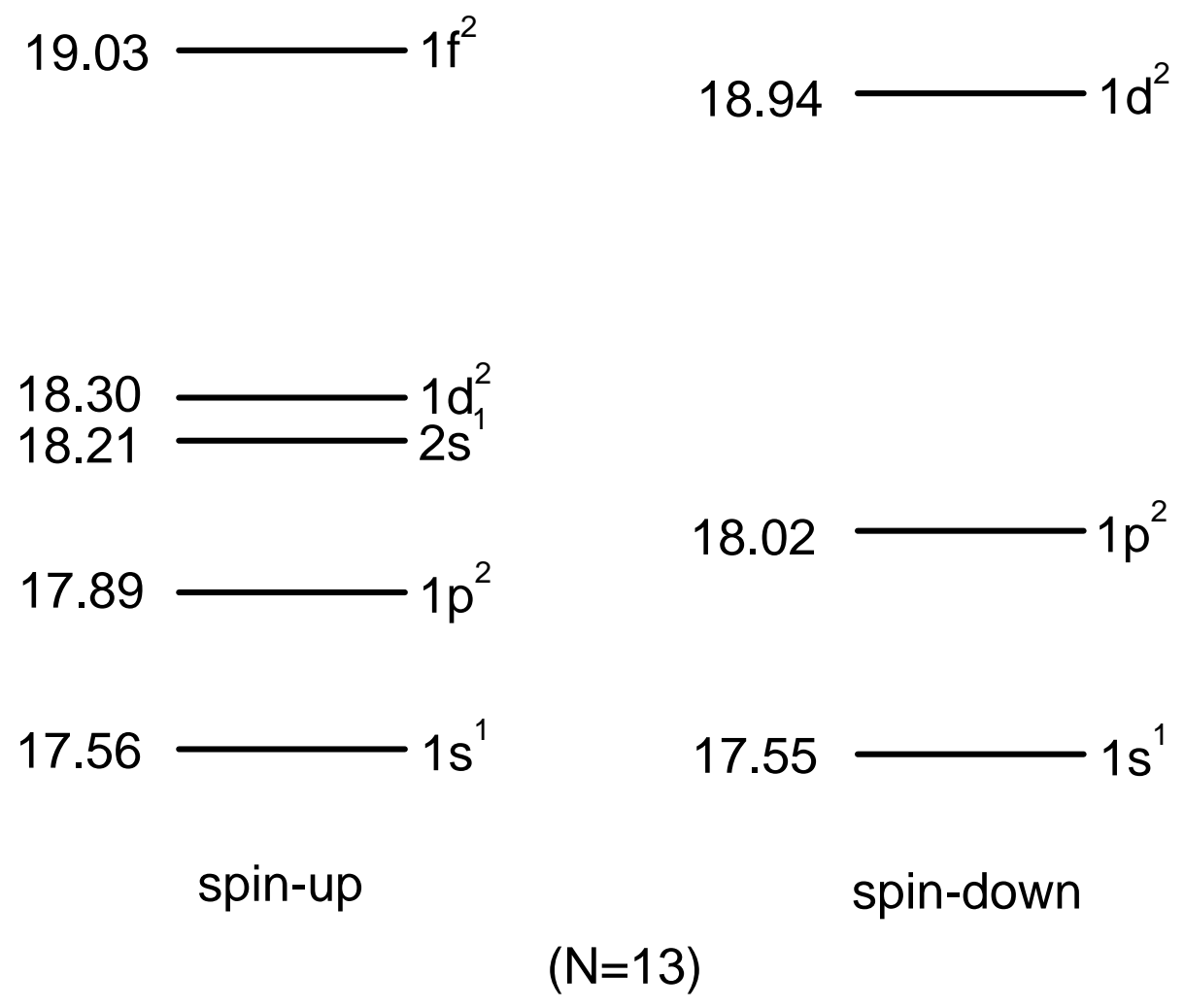

(b) 